ORIGINAL ARTICLE

\title{
Metaplastic carcinoma of the breast: a clinicopathological review
}

\author{
G M Tse, P H Tan, T C Putti, P C W Lui, B Chaiwun, B K B Law
}

See end of article for authors' affiliations .....................

Correspondence to: Gary M Tse, Department of Anatomical and Cellular Pathology, Prince of Wales Hospital, Ngan Shing Street, Shatin, NT, Hong Kong; garyłse@cuhk.edu.hk

Accepted for publication 27 July 2005

Published Online First 7 February 2006
Background: Mammary metaplastic carcinoma encompasses epithelial-only carcinoma (high-grade adenosquamous carcinoma or pure squamous cell carcinoma), biphasic epithelial and sarcomatoid carcinoma and monophasic spindle cell carcinoma.

Aim: To evaluate the clinicopathological features of a large series of 34 metaplastic carcinomas.

Methods: 10 epithelial-only, 14 biphasic and 10 monophasic metaplastic carcinomas were assessed for nuclear grade, hormone receptor status, HER2/neu (cerbB2) oncogene expression, Ki-67 and p53, lymph node status and recurrence on follow-up.

Results: Intermediate to high nuclear grade were assessed in most (33/34) tumours. Oestrogen and progesterone receptors were negative in 8 of 10 epithelial-only, all 14 biphasic, and 9 of 10 monophasic tumours, cerbB2 was negative in 7 of 10 epithelial-only, all 14 biphasic and 8 of 10 monophasic tumours. Ki67 was found to be positive in 6 of 10 epithelial-only, 6 of 14 biphasic, and 7 of 10 monophasic tumours, whereas p53 was positive in 6 of 10 epithelial-only, 7 of 14 biphasic, and 8 of 10 monophasic tumours. Lymph node metastases were seen in 7 of 7 epithelial-only, 7 of 11 biphasic, and 3 of 7 monophasic tumours. Recurrences were seen in 4 of 7 epithelial-only, 8 of 9 biphasic, and 4 of 9 monophasic tumours.

Conclusions: All three subtypes of metaplastic carcinoma are known to behave aggressively, and should be differentiated from the low-grade fibromatosis-like metaplastic carcinoma, which does not metastasise. Oncological treatment options may be limited by the frequently negative status of hormonal receptor and cerbB2.
M etaplastic carcinoma of the breast denotes a heterogeneous group of uncommon malignant entities. This term is used by many to denote tumours with mixed epithelial and sarcomatoid components, as well as primary squamous or mixed adenocarcinoma and squamous cell carcinoma (SCC; fig 1).

In those cases with a sarcomatoid component the tumour cells can be classified into monophasic, composed of spindle cells only, or biphasic, being admixed with a carcinomatous (either ductal carcinoma or SCC) component. ${ }^{1}$ Other authors have systematically grouped the tumours into various diagnostic labels based on the presence of heterologous elements or osteoclastic giant cells, or on whether the tumour cells are monophasic, biphasic or squamoid. ${ }^{2-6}$ More recent literature presents cases with sarcomatoid components, with or without accompanying carcinomas. Several large series have evaluated as many as 24 cases, with particular emphasis on the clinical outcome $\mathrm{e}^{7-9}$ and the role of immunohistochemistry in the differentiation of this lesion from other disease entities, including phyllodes tumours and primary mammary sarcomas. ${ }^{10}{ }^{11}$ Metaplastic carcinoma is generally considered to be high grade, with clinical features similar to those of infiltrating duct carcinoma (IDC), although it may rarely present as inflammatory carcinoma. ${ }^{12}$ Recently, a low-grade variant, the fibromatosis-like group, has been reported, with a better outcome and mostly local recurrences but not distant metastases. ${ }^{13-15}$ SCC, and mixed adenocarcinoma and SCC have scarcely been dealt with in the literature in terms of outcome. In this study, a series of metaplastic carcinomas, including tumours from all three categories, are assessed and the clinical and pathological parameters reported.

MATERIALS AND METHODS

The histopathology files from five institutions were searched for metaplastic carcinoma of the breast. All the cases were formalin-fixed and routinely processed. All the histological slides were retrieved and reviewed, and the diagnosis was confirmed. Each tumour was assessed for three components:

- Adenocarcinoma component, which morphologically showed glandular or tubule formation or the presence of intracellular or extracellular mucin secretion

- Squamous component, when the cells showed characteristic polygonal appearance with a moderate amount of eosinophilic cytoplasm, with identifiable intercellular bridges, with or without keratin pearl formation

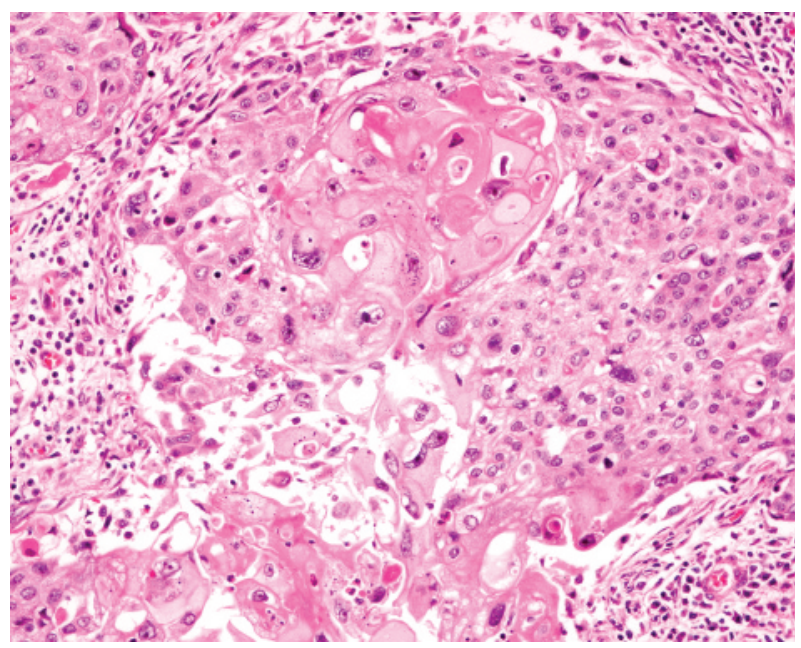

Figure 1 Squamous cell carcinoma of the breast. Haematoxylin and eosin staining, $\times 200$. 


\begin{tabular}{|c|c|c|c|}
\hline & Epithelial only $(n=10)$ & Biphasic $(n=14)$ & Monophasic $(n=10)$ \\
\hline Subtypes (n) & $\begin{array}{l}2 \text { adenosquamous } \\
8 \mathrm{SCC}\end{array}$ & $\begin{array}{l}10 \mathrm{IDC}+\text { sarcoma } \\
4 \mathrm{SCC}+\text { sarcoma }\end{array}$ & \\
\hline \multicolumn{4}{|l|}{ Age (years) } \\
\hline Range & $42-86$ & $43-79$ & $43-76$ \\
\hline Mean & 56 & 58 & 54 \\
\hline Median & 49 & 57 & 50 \\
\hline \multicolumn{4}{|l|}{ Tumour size $(\mathrm{cm})$} \\
\hline Range & $0.5-7$ & $2-11.5$ & $1.3-10$ \\
\hline Mean & 4.3 & 5.1 & 4 \\
\hline Median & 3.5 & 5 & 3 \\
\hline \multicolumn{4}{|l|}{ Nuclear grade (n) } \\
\hline High & 2 & 10 & 8 \\
\hline Intermediate & 6 & 4 & 2 \\
\hline Low & 1 & 0 & 0 \\
\hline $\begin{array}{l}\text { Oesotrogen } \\
\text { receptor }(\mathrm{n})\end{array}$ & 3 & 0 & 1 \\
\hline $\begin{array}{l}\text { Progesterone } \\
\text { receptor (n) }\end{array}$ & 2 & 0 & 1 \\
\hline cerbB2 (n) & 3 & 0 & 2 \\
\hline Ki-67 $(>10 \%, n)$ & 6 & 6 & 7 \\
\hline p53 $(>10 \%, n)$ & 6 & 7 & 8 \\
\hline
\end{tabular}

- Sarcomatoid or spindle cell areas, with the cells forming poorly cohesive sheets or with spindle cell morphology.

The sarcomatoid or solid component, when present alone, had to coexpress cytokeratin (AE1/AE3 or CAM5.2 or both) and vimentin, as shown by immunohistochemistry (AEl/AE3 (Dako, Glostrup, Denmark; 1:300), CAM5.2 (Becton Dickenson, San Jose, California, USA; 1:70) and vimentin (Dako; 1:2000)).

All the mammary metaplastic carcinomas were divided into three groups:

- Epithelial, with the tumour expressing both adenocarcinoma and SCC, or SCC alone

- Biphasic, with the tumour expressing the carcinoma component (either adenocarcinoma or SCC) and sarcomatoid or spindle cell component

- Monophasic, with the tumour being formed exclusively by the sarcomatoid or spindle cell component.

For each component, the tumour was assessed for the nuclear grade as $1-3$, indicating mildly, moderately or highly pleomorphic nuclear morphology. Immunohistochemistry was carried out on a representative section on all cases for the following antibodies by using the avidin-biotin method with microwave antigen retrieval. The following parameters were assessed by immunohistochemistry:

1. Oestrogen receptor (Neomarkers, Fremont, California, USA; $1: 400$ )

2. Progesterone receptor (Neomarkers; 1:300)

3. HER2/neu (cerbB2; Dako; 1:500)

4. Proliferation marker Ki-67 (MIBl; Dako; 1:100)

5. p53 (Dako; 1:40)

For oestrogen and progesterone receptors, moderate to strong nuclear staining of $10 \%$ or more of the tumour cells was considered to be positive and for cerbB2, moderate to strong complete membrane staining of $10 \%$ or more of the tumour cells was considered to be positive. MIBl and p53 staining was expressed as a percentage of cells showing moderate to strong nuclear staining, and was considered to be positive if the expression was $>10 \%$.

The medical records of the patients were reviewed, with emphasis on lymph node status at the time of surgery, if

\begin{tabular}{|c|c|c|c|}
\hline & Epithelial only $(n=10)$ & Biphasic $(n=14)$ & $\begin{array}{l}\text { Monophasic } \\
(\mathrm{n}=10)\end{array}$ \\
\hline Subtypes (n) & $\begin{array}{l}2 \text { adenosquamous } \\
8 \mathrm{SCC}\end{array}$ & $\begin{array}{l}10 \mathrm{IDC}+\text { sarcoma } \\
4 \mathrm{SCC}+\text { sarcoma }\end{array}$ & \\
\hline With AD & 7 & 11 & 7 \\
\hline With metastasis & 7 & 4 & 3 \\
\hline Morphology of metastases (n) & $\begin{array}{l}2 \text { adenosquamous } \\
5 \text { squamous or poorly } \\
\text { differentiated }\end{array}$ & $\begin{array}{l}3 \text { carcinoma only } \\
1 \text { carcinoma and sarcoma }\end{array}$ & \\
\hline $\begin{array}{l}\text { With follow-up } \\
\text { Follow-up time (months) }\end{array}$ & 7 & 9 & 7 \\
\hline Mean & 19 & 27 & 36 \\
\hline Median & 24 & 12 & 24 \\
\hline Died of disease (n) & 2 & 5 & 1 \\
\hline Died of other causes (n) & 0 & 1 & 2 \\
\hline Alive with disease (n) & 2 & 3 & 2 \\
\hline Alive without disease (n) & 3 & 0 & 4 \\
\hline
\end{tabular}


performed, and the follow-up duration and outcomewhether the patient was alive or dead, and if deceased, whether the death was related to the breast tumour.

\section{RESULTS}

A total of 34 metaplastic carcinomas were included in this report. These included 10 cases showing epithelial phenotype only, with 2 cases of mixed adenocarcinoma and SCC; and 8 cases of pure SCC. Of the 14 cases with biphasic phenotype showing mixed epithelial and sarcomatoid or spindle cell phenotypes, 10 cases had adenocarcinoma (all IDCs) and the remaining four cases had SCC as the epithelial component. The remaining 10 cases showed a monophasic pattern composed entirely of sarcomatoid or spindle cells with a phenotypic coexpression of epithelial and mesenchymal markers by immunohistochemistry.

Table 1 shows the patients' age, tumour size and histomorphological characteristics, and the immunohistochemistry results for each of these groups. Table 2 lists the axillary lymph node status and the clinical outcome. Of the patients who were alive but had recurrent disease, two were from the epithelial-only group, with one having brain metastasis ( 1 year) and the other having contralateral highgrade ductal carcinoma (1 year). Of the three patients from the biphasic group, who were alive with disease, two had recurrences ( 1 and 3 years, respectively) and one had brain metastasis ( 1 year). Of the two patients from the monophasic group, who were alive with disease, one had recurrence ( 1 year) and one had lymph node metastasis ( 3 years).

\section{DISCUSSION}

Metaplastic carcinoma is a rare malignancy of the breast, characterised by its high-grade nature. In this report, a large series of 34 cases was assembled, and we evaluated the biological parameters and clinical outcome between the squamoid carcinomas and the sarcomatoid or spindle cell carcinomas (fig 2) for similarity.

Most metaplastic carcinomas are sporadic, but there may be a slight propensity for metaplastic spindle cell carcinoma to arise from pre-existing lesions, including papillomas, complex sclerosing lesions and nipple adenomas. ${ }^{16} 17$ Metaplastic or spindle cell carcinomas arising from such lesions can show a varying degree of malignancy, ranging from low to high grade. This type of lesion should also be differentiated from the so-called reactive spindle cell nodule,

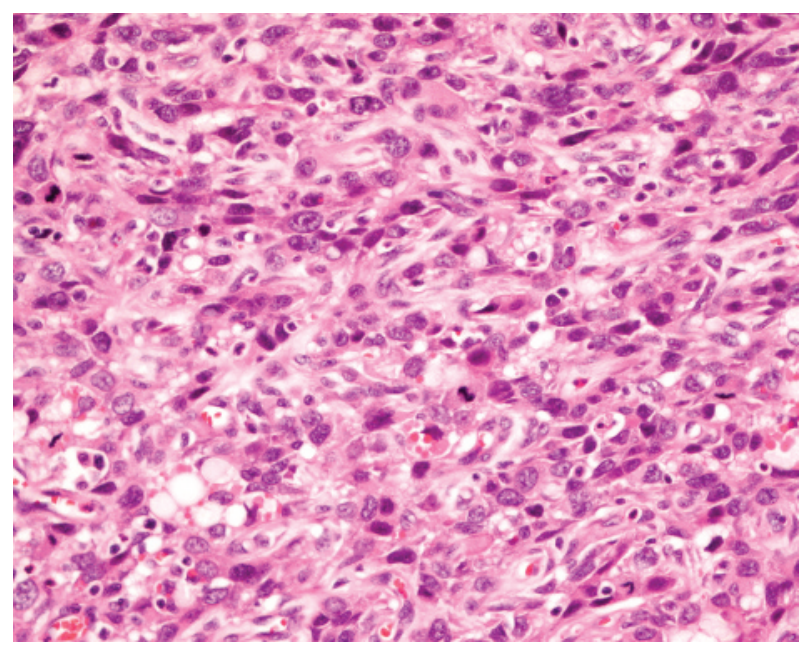

Figure 2 Spindle cell carcinoma, with the tumour cells showing plump and pleomorphic nuclei and brisk mitotic activity. Haematoxylin and eosin staining, $\times 200$. which is believed to be benign and may also complicate preexisting lesions such as papillomas or complex sclerosing lesions. ${ }^{18}$

In this series of 34 sporadic metaplastic carcinomas, we identified 24 cases of metaplastic carcinoma with a sarcomatoid or spindle cell component, with $14(58 \%)$ of the 24 cases being biphasic and $10(42 \%)$ of the 14 cases being monophasic. This is essentially in agreement with the rates of $11-33 \%$ reported in the literature. ${ }^{3} 101319$

In all the cases, levels of hormone receptors (oestrogen and progesterone receptors) and expression of the cerbB2 oncogene were low, with oestrogen and progesterone receptors being positive in only $4(12 \%)$ and $3(9 \%)$ of the 34 cases, respectively. Expression of the cerbB2 oncogene was also low, being only positive in 5 (15\%) of the 34 cases. The generally low percentage of expression of the hormone receptors is in agreement with the $0-25 \%$ reported in the literature. ${ }^{9}{ }^{12}$ Expression of cerbB2 warrants further discussion, as a low cerbB2 expression had been reported in metaplastic carcinoma. In our series of biphasic and monophasic tumours, only $2(8 \%)$ of the 24 cases were positive for cerbB2; an even lower figure of $0 \%$ had been reported $^{9}$ in the literature. This is in contrast with the general impression that in high-grade (grade 3 ) breast carcinomas, the cerbB2 positivity rate is about $35 \%,{ }^{20}{ }^{21}$ thus suggesting that metaplastic carcinoma may be biologically different from high-grade IDC.

The expression of Ki-67 and p53 among the three groups of metaplastic carcinomas in our series, with a $10 \%$ positivity as the cut-off, was high, with $6(60 \%)$ of 10 cases, $6(43 \%)$ of 14 cases and $7(70 \%)$ of 10 cases, respectively, showing $>10 \%$ positivity for epithelial-only, biphasic and monophasic metaplastic carcinomas. For p53, >10\% staining was seen in $4(40 \%)$ of 10 cases of epithelial-only carcinomas, $6(43 \%)$ of 14 cases of biphasic carcinomas and $8(80 \%)$ of 10 cases of monophasic metaplastic carcinomas. Although the expression of p53 and Ki-67 has not been extensively reported for metaplastic carcinoma of the breast, one sporadic case report noted the expression of these markers. ${ }^{22}$ Another report on a series of 24 low-grade spindle cell carcinoma of the breast showed that all the cases were $<5 \%$ positive for Ki-67, ${ }^{14}$ in stark contrast to the observation in this case series. This provides strong evidence for the high grade and aggressive nature of metaplastic carcinoma in general, irrespective of the histological subtypes-whether epithelial-only, biphasic or monophasic. The biological behaviour of the low-grade spindle cell carcinoma is probably distinct, and it would be prudent to consider this as a separate entity.

Further evidence of the aggressive nature of all the histological subtypes is apparent from the lymph node status and the follow-up result of the current cohort. Of the 25 patients who had known, histologically proven axillary status at the time of surgery $14(56 \%)$ patients showed positive axillary lymph node metastases. This is in contrast with the reported incidence of axillary metastasis of $8 \%$ in one series, ${ }^{8}$ but the figure in our study was similar to the $40 \%$ of another series. ${ }^{9}$ This again contrasts with the reported results of the low-grade spindle cell carcinoma, with the two large series reporting the absence of axillary metastases in all cases. ${ }^{13} 14$ The morphology of the metastases in most situations recapitulates that of the original tumour. For the epithelialonly and the monophasic carcinomas, this poses no diagnostic problem in identifying metastasis. In the current cohort, the two adenosquamous carcinomas showed adenosquamous components in the metastases, and the SCC showed squamous or poorly-differentiated carcinoma in the metastases. Problems may, however, arise for the biphasic carcinoma. In the four cases with metastases, three showed a carcinoma component only in the metastases, and in only 

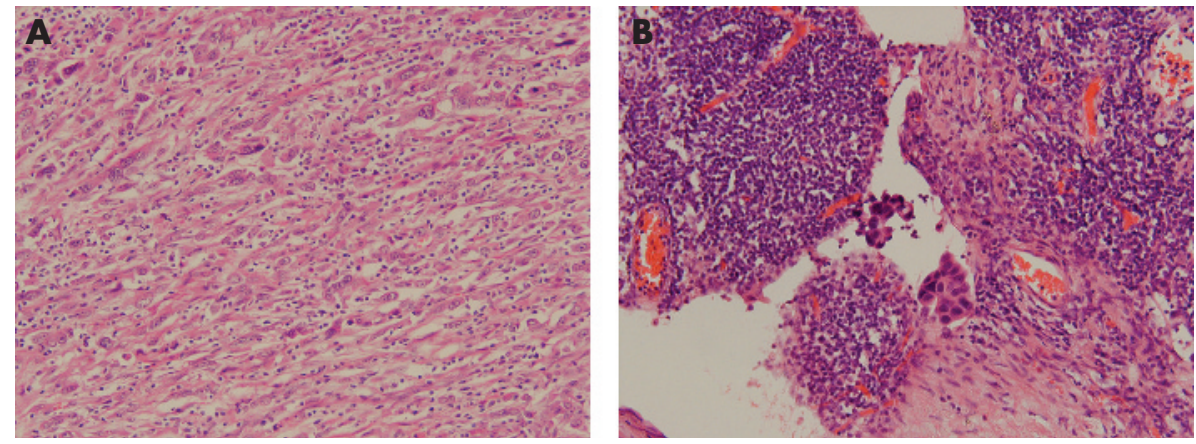

Figure 3 (A) Monophasic sarcomatoid metaplastic breast carcinoma, featuring elongated plump cells with enlarged pleomorphic vesicular nuclei and distinct nucleoli. Admixed inflammatory cells are seen. Haematoxylin and eosin (H\&E) staining, $\times 100$. (B) Corresponding lymph node metastasis, showing tight cohesive clusters of malignant epithelial cells within the nodal sinuses. H\&E, $\times 400$.

one case was the metastasis biphasic. Furthermore, in one case, the metastasis seemed to be more epithelial than the primary tumour which was more spindled (figs 3A,B). Bearing this fact in mind is important, as failure to recognise the possible morphological discrepancy between the primary and metastasis may result in inappropriate treatment and investigations for a possible new primary erroneously perceived.

Follow-up data were available for 25 patients; 11 dead ( 14 alive). Among those who died, 8 (73\%) died of disease, and of the survivors, 7 (50\%) also had evidence of disease at the end of the follow-up period. Viewed from another perspective, of a total of 25 patients, $15(60 \%)$ had evidence of disease $(8$ died, 7 were alive). On comparing the parameters between these two groups, we found no differences between hormonal receptor status and the expression of cerbB2, Ki-67 and p53. The only difference was the percentage of positive axillary lymph node status, which was positive in $9(75 \%)$ of 12 patients with disease compared with $4(40 \%)$ of 10 patients from the disease-free group.

When we look at the individual subtype of metaplastic carcinomas, the percentage of patients with disease (dead or alive) are $4(57 \%)$ of 7 patients, $8(89 \%)$ of 9 patients and 3 $(33 \%)$ of 9 patients, respectively, from the groups of epithelial-only, biphasic and monophasic metaplastic carcinomas. As the number of cases in these categories is small, the significance of this if any, remains unclear. Although the diagnosis of the epithelial-only subtype (adenosquamous or

\section{Take-home messages}

- Metaplastic carcinoma encompasses tumours with mixed epithelial and sarcomatoid components, as well as primary squamous or mixed adenocarcinoma and squamous carcinoma; the mixed group can be further classified into monophasic (spindle cell only), or biphasic (mixed spindle and carcinoma cells).

- In all types of metaplastic carcinoma, hormone receptors (oestrogen, progesterone) and expression of cerbB2 oncogene are low $(<20 \%)$, and this may have an implication on treatment options.

- Diagnosing the biphasic and monophasic metaplastic carcinoma requires coexpression of cytokeratin and vimentin. $\mathrm{AE} 1 / \mathrm{AE} 3$ remains the most widely used and the most sensitive marker, together with vimentin, for defining the metaplastic spindle cells, particularly for the monophasic subtype. pure SCC) can be based only on morphological assessment, diagnosing the biphasic and monophasic types of metaplastic carcinoma requires the use of immunohistochemistry. Cytokeratin and vimentin positivity is the defining feature. ${ }^{8}$ Some authors reported a rather low expression of cytokeratin markers (8/18 (44\%) cases for AE1/AE3, and 9/18 (50\%) cases for CAM5.2), but if both markers were combined, the pick-up rate increased to $10(55 \%)$ of 18 cases. Furthermore, the spindle cells were reported to show the highest percentage of expression for myoepithelial markers (34bE12, smooth muscle cell actin). ${ }^{8}$ Most other recent studies report a higher percentage of AEl/AE3 expression, ranging from 63\% to $100 \% .^{3671113}$ Hence, AEl/AE3 remains the most widely used and most sensitive marker, with vimentin, for defining the metaplastic spindle cells, particularly for the monophasic subtype.

In summary, we report a large series of metaplastic carcinomas, by conventional usage. The diagnosis of this group of lesions is by morphology for carcinomas with a squamous component or a pure squamous carcinoma, and by immunohistochemistry for those with a sarcomatoid or spindle cell carcinoma, coexpressing vimentin and cytokeratin. The combined use of AE1/AE3 and CAM5.2 shows a higher sensitivity than each marker alone. All three subtypes may have aggressive biological behaviour, as evidenced by the high percentage of lymph node metastases at the time of diagnosis and the rather dismal outcome, as well as the high p53 and Ki-67 index. The absence of hormonal receptors and cerbB2 oncoprotein expression may further limit oncological treatment options.

\section{Authors' affiliations}

G M Tse, Department of Anatomical and Cellular Pathology, Prince of Wales Hospital, Chinese University of Hong Kong, Hong Kong P H Tan, Department of Pathology, Singapore General Hospital, Singapore

T C Putti, Department of Pathology, National University Hospital, National University of Singapore, Singapore

P C W Lui, Department of Pathology, United Christian Hospital, Hong Kong

B Chaiwun, Department of Pathology, Chiang Mai University, Chiang Mai, Thailand

B K B Law, Breast Center, Union Hospital, Hong Kong

Competing interests: None declared.

\section{REFERENCE}

1 Elston CW, Ellis IO. The breast. In:Systemic pathology.Vol.13.3rd edn. Edinburgh: Churchill Livingstone, 1998:323-31.

2 Wargotz ES, Norris HJ. Metaplastic carcinomas of the breast. I. Matrixproducing carcinoma. Hum Pathol 1989;20:628-35.

3 Wargotz ES, Deos PH, Norris HJ. Metaplastic carcinomas of the breast. II. Spindle cell carcinoma. Hum Pathol 1989;20:732-40. 
4 Wargotz ES, Norris HJ. Metaplastic carcinomas of the breast. III. Carcinosarcoma. Cancer 1989;64:1490-9.

5 Wargotz ES, Norris HJ. Metaplastic carcinomas of the breast. IV. Squamous cell carcinoma of ductal origin. Cancer 1990;65:272-6.

6 Wargotz ES, Norris HJ. Metaplastic carcinomas of the breast: V. Metaplastic carcinoma with osteoclastic giant cells. Hum Pathol 1990;21:1142-50.

7 Christensen L, Schiodt T, Blichert-Toft M. Sarcomatoid tumours of the breast in Denmark from 1977 to 1987. A clinicopathological and immunohistochemical study of 100 cases. Eur J Cancer 1993;29A:1824-31.

8 Kurian KM, Al-Nafussi A. Sarcomatoid/metaplastic carcinoma of the breast: a clinicopathological study of 12 cases. Histopathology 2002;40:58-64.

9 Khan HN, Wyld L, Dunne B, et al. Spindle cell carcinoma of the breast: a case series of a rare histological subtype. Eur J Surg Oncol 2003;29:600-3.

10 Dunne B, Lee AH, Pinder SE, et al. An immunohistochemical study of metaplastic spindle cell carcinoma, phyllodes tumour and fibromatosis of the breast. Hum Pathol 2003;34:1009-15.

11 Adem C, Reynolds C, Adlakha H, et al. Wide spectrum screening keratin as a marker of metaplastic spindle cell carcinoma of the breast: an immunohistochemical study of 24 patients. Histopathology 2002;40:556-62.

12 Kuo SH, Chen CL, Huang CS, et al. Metaplastic carcinoma of the breast: analysis of eight Asian patients with special emphasis on two unusual cases presenting with inflammatory-type breast cancer. Anticancer Res 2000;20:2219-22.

13 Gobbi H, Simpson JF, Borowsky A, et al. Metaplastic breast tumours with a dominant fibromatosis-like phenotype have a high risk of local recurrence. Cancer 1999;85:2170-82.
14 Sneige N, Yaziji H, Mandavilli SR, et al. Low-grade (fibromatosis-like) spindle cell carcinoma of the breast. Am J Surg Pathol 2001;25:1009-16.

15 Al-Bozom IA, Abrams J. Spindle cell carcinoma of the breast, a mimicker of benign lesions: case report and review of the literature. Arch Pathol Lab Med 1996; 120:1066-8.

16 Gobbi H, Simpson JF, Jensen RA, et al. Metaplastic spindle cell breast tumours arising within papillomas, complex sclerosing lesions, and nipple adenomas. Mod Pathol 2003;16:893-901.

17 Denley H, Pinder SE, Tan PH, et al. Metaplastic carcinoma of the breast arising within complex sclerosing lesion: a report of five cases. Histopathology 2000;36:203-9.

18 Gobbi H, Tse G, Page DL, et al. Reactive spindle cell nodules of the breast after core biopsy or fine-needle aspiration. Am J Clin Pathol 2000;113:288-94.

19 Ostrowski JL, Horgan K, Krausz T, et al. Monophasic sarcomatoid carcinoma of the breast. Histopathology 1998;32:184-6.

20 Quenel N, Wafflart J, Bonichon F, et al. The prognostic value of c-erbB2 in primary breast carcinomas: a study on 942 cases. Breast Cancer Res Treat 1995:35:283-91.

21 Ridolfi RL, Jamehdor MR, Arber JM. HER-2/neu testing in breast carcinoma: a combined immunohistochemical and fluorescence in situ hybridization approach. Mod Pathol 2000;13:866-73.

22 Nakayama $Y$, Iwasaki $\mathrm{H}$, Iwanaga $\mathrm{S}$, et al. Spindle cell carcinoma of the breast: a case report and an immunohistochemical study including p53 and Ki-67 expression. Pathol Int 1997;47:404-11. 